ISSN 2693-2504

\title{
Effect of Aspirin on Experimental Atherosclerosis in Rabbits Submitted To Hypercholesterolemic Diet
}

Journal of Bioscience \& Biomedical Engineering

Research Article

Ieda Edite Lanzarini Lopes ${ }^{1}$, Ricardo Luiz Smith ${ }^{2}$, Stanley Nigro ${ }^{2}$, Silvia S M Ihara ${ }^{2}$, Leonor do Espírito Santo de Almeida Pinto ${ }^{2}$, Anita L R Saldanha ${ }^{1}$ and Tania Leme da Rocha Martinez ${ }^{{ }^{*}}$

${ }^{1}$ Nephrology Department, BP - A Beneficencia Portuguesa de São Paulo, São Paulo, Brazil

${ }^{2}$ Department of Pathology - UNIFESP - Universidade Federal de São Paulo, São Paulo, Brazil

\section{*Correspondence authors}

Tania Leme da Rocha Martinez

BP - A Beneficência Portuguesa de São Paulo

Rua Comandante Ismael Guilherme

358 - Jardim Lusitânia 04031-120 - São Paulo - SP

Brazil

\begin{abstract}
Aspirin, besides its antithrombotic activity, has also been quoted for protective effect reducing new coronary lesions.

Objective: Considering the growing interest in the study of drugs that prevent the progression of atherosclerotic lesion, the objective of this study was to determine the effect of aspirin in experimental atherogenesis induced in rabbits fed with cholesterol rich diets.
\end{abstract}

Method: Thirty adult, New Zealand white male rabbits, with $3.4 \mathrm{~kg}$ of body weight were submitted to a $1.5 \%$ cholesterol-rich-diet for 9 weeks. Aspirin was triturated, mixed with chloroform and incorporated to the normal chow. The drug was given to the rabbits every day in portions of $20 \mathrm{~g}$ of chow with $100 \mathrm{mg}$ of aspirin. The rabbits were divided in 3 groups as follows: A) Cholesterol-rich-diet $(n=10) ; B)$ Cholesterol-rich-diet plus aspirin ( $n=10)$ and C) Normal chow $(n=10)$. Blood samples were collected before starting the diet, at 5 weeks and at sacrifice (9 weeks) for determination of total cholesterol, triglycerides, enzymes and hematological tests. After sacrifice, staining of the aorta was done by Sudan III for visualization of sudanophillic plaques. The percentual of aorta couvered with lipidic deposits were determined by computerized planimetry.

Results: Total cholesterol ( $\mathrm{mg} / \mathrm{dl})$ was per group: $A-T O=52, T 5=424$ and $T 9=1.483 ; B-T O=32, T 5=755$ and $T 9=1.436$; $C-T O=41, T 5=22$ and $T 9=27$. Planimetry data did not differ among groups $A(23.3 \%)$ and $B(27.3 \%)$. Scanning microscopy - the interpretation of the document images in three groups showed insignificant platelet deposition in all aortic segments both in groups $B$ and $C$.

Conclusion: This experiment enhances the theoretic basis for the protective effect of aspirin as an antiaggregant factor in the experimental aortic atherosclerotic lesion.

Keywords : Antiaggregant, Aspirin, Atherosclerosis, Cholesterol Rich Diet.

\section{Abbreviations}

\section{SEM: Scanning Electron Microscopy}

\section{Introduction}

Numerous clinical studies already described in the literature clearly demonstrate the correlation between the use of antiplatelet agents, especially aspirin and the frequency reduction of clinical events resulting from coronary artery disease. Thus, aspirin is currently being used as complementary therapy in acute ischemic syndromes such as unstable angina and acute myocardial infarction, in order to prevent possible complications resulting from atherosclerotic processes.

Aspirin is still the main and most affordable drug studied in the literature regarding prevention of cardiovascular disease, in spite of all the works published on anticoagulants $[1,2,3]$.
Analyzing the beneficial effects of aspirin in clinical studies, in particular acute myocardial infarction and unstable angina, we can conclude that they are due to its effects on platelet aggregation and subsequent vascular occlusion, preventing vasospasm or both. There may, however, be other mechanisms related to thrombosis, leading to infarction or death. Thrombin itself is extremely thrombogenic and aggregating, and this activity is not inhibited by aspirin. In fact, platelets exposed to aspirin can still cause an aggregating reaction, despite the suppression of thromboxane A2 synthesis, because interactions between platelets and vessel wall, von Willebrand factor and fibrinogen, can happen independently of aspirin that acts on platelet aggregability via thromboxane A2.

The tendency to thrombosis is also related to the degree of stenosis and arterial injury. High parietal stress at the level of a 
critical coronary stenosis contributes to platelet adhering and aggregation by a mechanism independent of cyclooxygenase [4].

Aspirin inhibits thrombosis only if thromboxane A2 formation by platelets plays a major role in thrombus growth: its effect is small when thrombin generation and fibrin formation are dominant factors in thrombosis. Aspirin does not seem to be effective in inhibiting the adhesion of a simple layer of platelets to the injured endothelium, but may prevent additional platelet aggregation, being important in limiting the extent of this aggregation [5].

\section{Objective}

In our study we analyzed the effect of aspirin on the development of atherosclerotic lesions in the aortas of rabbits made hyperlipidemic through a high-cholesterol diet (1.5\%).

\section{Method}

We used the acute induction model standardized by the Experimental Atherosclerosis team of the Lipid Sector of the Federal University of São Paulo, in an adaptation of the works of Badimon et al. (1990) [6].

The most frequent studies with an animal model of induction of hypercholesterolemia through diet, used concentrations between $0.5 \%$ and $1.5 \%$ of cholesterol, for periods of nine weeks to three months, verifying extremely high cholesterol levels $[7,8]$ Although under non-physiological conditions, this model has been shown to be very useful for the study of the different mechanisms involved in atherosclerosis, besides enabling therapeutic and etiopathogenic approaches in the study of disease progression and regression.

We opted for the acute model with cholesterol concentration at $1.5 \%$ at nine weeks, since Thomaz (1992) [8], administering this same diet, verified a large number of lesions in a shorter time (five and nine weeks), when compared with the $0.5 \%$ diet used in the Cotrim (1991) [7] study, which obtained atheromatous lesion only after 90 days (twelve weeks).

We complement the study of the effect of aspirin on experimental atherosclerosis, analyzing the changes in the endothelium of the aortas through Scanning Electron Microscopy (SEM) to try to visualize through this technique, the antiaggregating action platelet of aspirin, inducing changes in the development of atherosclerotic plaques.

The SEM technique has been used to examine ultrastructural changes of the endothelial surface, platelet interactions with the endothelium and the effect of antiplatelet agents, thrombotic processes and the development of atherosclerosis.

\section{Results}

Serum cholesterol levels increased rapidly in rabbits on a hypercholesterolemic diet, reaching extremely high values in relation to the basal level of the animal $(43 \mathrm{mg} / \mathrm{dl})$, due to the slow excretion of exogenous cholesterol by the rabbit, when submitted to a high-cholesterol diet $[9,10]$.

After 9 weeks of hypercholesterolemic diet, serum cholesterol levels of $1,483 \mathrm{mg} / \mathrm{dl}$ and $1,436 \mathrm{mg} / \mathrm{dl}$, respectively, in the two hypercholesterolemic groups treated with aspirin or not, which were close to those obtained by Badimon et al. (1990) [6], with serum cholesterol levels of $1,559 \mathrm{mg} / \mathrm{dl}$ in rabbits submitted to a $0.5 \%$ cholesterol diet for 90 days. Cotrim (1991)[7], with the same concentration, reached $1,342 \mathrm{mg} / \mathrm{dl}$ in 60 days and $1,089 \mathrm{mg} / \mathrm{dl}$ in 90 days. Thomaz (1992) [8], with a diet of $1.5 \%$, reached levels of $1,469 \mathrm{mg} / \mathrm{dl}$ in nine weeks and Sun et al. (1993) [11], in twelve weeks, with a $0.3 \%$ diet, obtained cholesterol levels of $1,080 \mathrm{mg} / \mathrm{dl}$.

In the triglyceride dosages, we verified an increase in the results of the groups submitted to atherogenic diet, probably due to the technique used, which used automated methodology in methodology where the turbidity of sera due to the high intake of cholesterol may have caused this interference.

As for enzyme dosages, which indirectly evaluate aspects of liver physiology, the results of liver function tests demonstrate significant but discrete alterations in the treated group, possibly explained by the overload in intracellular metabolism of the hepatocyte with hypercholesterolemiante diet plus aspirin.

In order to monitor the general evolution of the animals, hematological measurements (haematocrit, hemoglobin, white blood cells and platelets) were made, which reflected a certain degree of variability in laboratory measurements. Despite these variations in individual dosages or even in the comparison between groups, these values did not reach levels of abnormalities that could compromise the experiment.

We verified a significant decrease in haematocrit and hemoglobin levels, a fact also reported by Stehbens (1986) [12], which can be attributed to lower feed intake in groups submitted to atherogenic diet. Even so, the animals did not present weight changes. We also observed an increase in white blood cells in the three groups studied, probably in response to the aggressions resulting from the cloistered conditions to which the animals were submitted. When analyzing the count of platelet numbers, we did not observe significant differences.

In the microscopic study of the aortas, we observed plaques with morphological characteristics of the eccentric type with intense lipid content and presence of numerous foamy cells in all samples of hypercholesterolemic animals. We verified formation of lesions of the type of fatty striae, without ulceration or development of complicated plaques.

In addition to the thoracic aorta presenting a higher number of lesions, through microscopic observation, we found that the atheromatous plaques in this region had a higher height than those of the abdominal region. Comparing, however, the morphometric determinations of the thickness of the lesions, in the hypercholesterolemic groups treated or not, we did not find significant difference, similarly to the evaluations of the area of the injured aorta. 
In our studies, we observed a predominance of hydropic degeneration in hepatocytes of groups submitted to hypercholesterolemic diet, being more pronounced in group A (70\% of moderate degeneration and $10 \%$ of intense degeneration) than in group B (30\% of moderate degeneration and $70 \%$ of mild degeneration). In the control group, fed only with normal diet, we observed the liver with preserved hepatocytes without histological alterations.

Hepatocytic macrovascular steatosis occurred in rare cases, with mild intensity and only in groups with atherogenic diet (30\% in group A and $10 \%$ in group B). We recorded the presence of parenchydate inflammatory infiltrates and portal in samples of all groups, even in animals of the group with normal diet $(20 \%$ parenchyma and $30 \%$ portal), which could not be related to the type of diet or treatment received, but to the response of the animals to the cloister conditions, or even to parasitosis. The frequency of inflammatory infiltrates, however, was higher in the groups with hypercholesterolemic diet, being slightly higher in the treated group $(60 \%$ and $50 \%$ of parenchymal and portal infiltrate in group A and $70 \%$ and $60 \%$ in group B), a result in agreement with the slightly increased serum levels of liver function tests.

In the analysis of the endothelial surface by SEM in the untreated hypercholesterolemic group, we verified an intense platelet aggregate, where it was possible to observe structural changes of platelets, including the emission of pseudopods, regions with extensive fibrin networks with some other blood elements, where the endothelial surface was interrupted or bulging probably by the presence of foamy cells inside. It was also possible to observe macrophages adherent to the surface.

\section{Discussion}

Stehbens (1986) [12], in an extensive review on animal model in atherosclerosis, reported that rabbits fed cholesterol for prolonged periods may exhibit weight loss, hair loss, hyperquethatosis in association with alopecia, eventually dying in a caquectic state. Our animals, however, did not present any of these alterations. These phenomena may not have occurred in our study, because we used an acute hypercholesterolemia induction model, performed in a short period of time (nine weeks).

In the rabbit developed hypercholesterolemic by diet, there are no atherosclerotic lesions with the same characteristics as those found in man. Ulcerations, thrombosis or aneurysms are not observed on the intimal surface of the aorta of these rabbits, although compromised. The high-cholesterol diet in rabbits leads to the formation of lesions of the type fatty striae in which lipid deposition is observed, especially in the subintimal region $[12,13,14]$.

One of the ways to reproduce lesions closer to those of man is to associate other elements such as vegetable oils with the hypercholesterolemic diet $[15,16,17]$. As in our study we used only a normal rabbit diet plus pure cholesterol, the lesions that developed had a structure similar to that of fatty striae.
In our study, aiming to analyze the effect of aspirin on experimental atherosclerosis in hypercholesterolemic rabbits per diet, we used a dose of $100 \mathrm{mg} /$ day, which is the currently recommended dose for humans, equivalent in rabbits to $30 \mathrm{mg} /$ $\mathrm{kg}$ of weight. We chose this dose based on the experimental works of de Debons et al. (1981)[18] who used a dose of 100 $\mathrm{mg} / \mathrm{kg}$ and Sun et al. (1993) [11], which analyzed the effect of varying doses of $1 \mathrm{mg} / \mathrm{kg}, 10 \mathrm{mg} / \mathrm{kg}, 30 \mathrm{mg} / \mathrm{kg}$ and $60 \mathrm{mg} /$ $\mathrm{kg}$, on bleeding time, platelet aggregation and development of atherosclerotic lesions.

With the best knowledge of the mechanism of action of aspirin, which blocking cyclooxygenase acts by simultaneously inhibiting thromboxane A2 and prostacyclin, studies have been trying to find an ideal dose in which a thromboxane A2 inhibition produced by platelets would be inhibited with a less intense action in the production of prostascline produced by endothelial cells, so that , in the balance sheet, we would have much more phenomena of vasodilatation and platelet antiaggregation in relation to the opposite phenomena of vasoconstriction and aggregation[19]. Thus, lower doses or less frequent administration could give aspirin a more efficient effect.

Analyzing the effect of different doses of aspirin on the inhibition of thromboxane A2 production, studies have shown that with the dose of $80 \mathrm{mg}$, in addition to inhibiting the production of thromboxane A2, there is less action in inhibiting the production of prostacyclin [20]. At doses of $50 \mathrm{mg} /$ day, there was a higher inhibition in the formation of thromboxane A2 than prostacyclin in patients with atherosclerosis [21].

Better results can be obtained if aspirin is administered every other day [22,23], because when blocking the production of Thromboxane A2 by platelet, as this does not regenerate, a new platelet is needed to restart its production, which requires days, the same not occurring with the vascular endothelium, which recovers rapidly. If aspirin is then administered every other day, there would be no time for platelets to produce thromboxane $\mathrm{A} 2$, but rather for the production of prostacyclin through the vessel.

In the macroscopic analysis of aortic lesions, cordoned with SUDAM III, we observed marked lipid deposition in the intimal layer of the arteries in both hypercholesterolemic groups, with atherosclerotic lesions in greater number and intensity in the aortic arch region and thoracic aorta confirming the already widely established data. When the areas of the aorta compromised by atherosclerotic lesions were quantified by computerized planimetry, we verified great individual variation in the two treated and untreated hypercholesterolemic groups $(23.3 \%$ and $27.3 \%$ ), but there was no statistically significant difference between the groups regarding the percentage of injured area.

\section{Conclusion}

In our study, aspirin did not exert a protective effect on the installation of atherosclerotic lesions in rabbits with a hypercholesterolemic diet, however, we verified, through observation by SEM lower platelet aggregation, as well as lower frequency in 
fibrin findings in animals treated with the drug. These observations reinforce the importance of the use of antiplatelet agents in the therapy of patients with coronary diseases, associated with hyperlipidemia, which present greater susceptibility of events resulting from complications of atherosclerotic plaque. In conclusion, we have made it very clear that further studies are needed to establish the real benefits of aspirin in the development and complications of atherosclerotic plaque, since its beneficial effect as an antiplatelet substance depends on the interrelationship of several mechanisms involved in thrombogenic and atherosclerotic processes. Thus, in addition to carefully presenting our steps during the process, we want them to be another stimulus for others to take it forward, in scientific advancement and improvement.

\section{Acknowledgments}

None.

\section{Conflicts of interest}

No conflict of interest.

\section{References}

1. Huang WY, Daugherty SE, Shiels MS, Purdue MP, Freedman ND, Abnet CC, Hollenbeck AR, Hayes RB, Silverman DT, Berndt SI (2018) Aspirin use and mortality in two contemporary US cohorts. Epidemiology. 2018;29(1):126133. doi: 10.1097/EDE.0000000000000746

2. Ittaman SV, VanWormer JJ, Rezkalla SH (2014) The role of aspirin in the prevention of cardiovascular disease. Clin Med Res. 2014;12(3-4):147-154. doi:10.3121/cmr.2013.1197

3. Van't Hof JR, Duval S, Walts A, Kopecky SL, Luepker RV, Hirsch AT (2017) Contemporary primary prevention aspirin use by cardiovascular disease risk: impact of US preventive services task force recommendations, 2007 2015: a serial, cross-sectional study. J Am Heart Assoc. 2017;6(10): e006328. doi: 10.1161/JAHA.117.006328

4. Shah PK, Forrester JS (1991) Pathophysiology of acute coronary syndromes. Am J Cardiol. 1991;68(12):16C-23C. doi: 10.1016/0002-9149(91)90219-b.

5. Cleland JG, Krikler DM (1993) Modification of atherosclerosis by agents that do not lower cholesterol. Br Heart J. 1993;69(1 Suppl): S54-62. doi: 10.1136/hrt.69.1 suppl. s54

6. Badimon JJ, Badimon L, Fuster V (1990) Regression of atherosclerotic lesions by high density lipoprotein plasma fraction in the cholesterol-fed rabbit. J Clin Invest. 1990;85(4):1234-1241. doi: 10.1172/JCI114558.

7. Cotrim FLS (1991) Induction of an experimental model of atherosclerosis in rabbits: biochemical and anatomopathological study, São Paulo, 1991. Dissertation (Master's degree in Morphology) - Federal University of São Paulo. https://search.bvsalud.org/unifesp/resources/index.php? $\mathrm{q}=\% 2 \mathrm{Bid}:(\% 22$ epm-009404\%22)\&lang=pt\&printMod$\mathrm{e}=$ true

8. Thomaz L (1992) Acute induction of experimental atherosclerosis in rabbits submitted to hypercholesterolemic diet]. São Paulo, 1992. Dissertation (Master's degree in
Medicine) - Federal University of São Paulo.

https://search.bvsalud.org/unifesp/resources/epm-010103

9. Armstrong ML, Heistad DD (1990) Animal models of atherosclerosis. Atherosclerosis. 1990;85(1):15-23. doi: 10.1016/0021-9150(90)90178-1

10. Wissler RW, Vesselinovitch D (1990) Can atherosclerotic plaques regress? Anatomic and biochemical evidence from nonhuman animal models. Am J Cardiol. 1990;65(12): 33F-40F. doi: 10.1016/0002-9149(90)91253-3

11. Sun YP, Zhu BQ, Sievers RE, Isenberg WM, Parmley WW (1993) Aspirin inhibits platelet activity but does not attenuate experimental atherosclerosis. Am Heart $J$. 1993;125(1): 79-86. doi: 10.1016/0002-8703(93)90059-i

12. Stehbens WE (1986) An appraisal of cholesterol feeding in experimental atherogenesis. Prog Cardiovasc Dis. 1986;29(2): 107-128. doi: 10.1016/0033-0620(86)900216

13. St Clair RW (1983) Atherosclerosis regression in animal models: current concepts of cellular and biochemical mechanisms. Prog Cardiovasc Dis. 1983;26(2):109-132. doi: 10.1016/0033-0620(83)90026-9

14. Vesselinovitch D (1988) Animal models and the study of atherosclerosis. Arch Pathol Lab Med. 1988;112(10):10111017. https://pubmed.ncbi.nlm.nih.gov/3052351/

15. Constantinides P, Booth J, Carlson G (1960) Production of advanced cholesterol atherosclerosis in the rabbit. Arch Pathol. 1960;70: 712-724. https://pubmed.ncbi.nlm.nih. gov/13695141/

16. Constantinides P (1961) Production of experimental atherosclerosis in animals. $J$ Atherosclerosis Res. 1961;1(5-6): 374-385. https://doi.org/10.1016/S03681319(61)80013-6.

17. Kritchevsky D, Tepper SA, Kim HK, Story JA, Vesselinovitch D, Wissler RW (1976) Experimental atherosclerosis in rabbits fed cholesterol-free diets: 5. Comparison of Peanut, Corn, Butter, and Coconut Oils. Exp Mol Pathol. 1976;24(3): 375-391. https://doi.org/10.1016/00144800(76)90072-1

18. Debons AF, Fani K, Jimenez FA (1981) Enhancement of experimental atherosclerosis by aspirin. J Toxicol Environ Health. 1981;8(5-6): 899-906. doi: $10.1080 / 15287398109530124$

19. Viinikka L (1990) Acetylsalicylic acid and the balance between prostacyclin and thromboxane A2. Scand J Clin Lab Invest Suppl. 1990;201: 103-108. doi: 10.3109/00365519009085806

20. Weksler BB, Pett SB, Alonso D, Richter RC, Stelzer P, Subramanian V, Tack-Goldman K, Gay WA Jr (1983) Differential inhibition by aspirin of vascular and platelet prostaglandin synthesis in atherosclerotic patients. $N$ Engl J Med. 1983;308(14): 800-805. doi: 10.1056/ NEJM198304073081402

21. Carlsson I, Benthin G, Petersson AS, Wennmalm A (1990) Differential inhibition of thromboxane A2 and prostacyclin synthesis by low dose acetylsalicylic acid in atherosclerotic patients. Thromb Res. 1990;57(3): 437-444. doi: 10.1016/0049-3848(90)90259-f 
22. Weksler BB, Tack-Goldman K, Subramanian VA, Gay WA Jr (1985) Cumulative inhibitory effect of low-dose aspirin on vascular prostacyclin and platelet thromboxane production in patients with atherosclerosis. Circulation. 1985;71(2): 332-340. doi: 10.1161/01.cir.71.2.332

23. Steering Committee of the Physicians' Health Study Research Group. Preliminary report: Findings from the aspirin component of the ongoing Physicians' Health Study. $N$ Engl J Med. 1988;318(4): 262-264. doi: 10.1056/NEJM198801283180431

Copyright: (C2021 Tania Leme da Rocha Martinez. This is an open-access article distributed under the terms of the Creative Commons Attribution License, which permits unrestricted use, distribution, and reproduction in anymedium, provided the original author and source are credited. 